\title{
PAPER \\ Comprehensive Performance Evaluation of Universal Time-Domain Windowed OFDM-Based LTE Downlink System
}

\author{
Keiichi MIZUTANI $^{\dagger \text { a)}}$, Takeshi MATSUMURA ${ }^{\dagger}$, Members, and Hiroshi HARADA ${ }^{\dagger}$, Fellow $^{\prime}$
}

\begin{abstract}
SUMMARY A variety of all-new systems such as a massive machine type communication (mMTC) system will be supported in $5 \mathrm{G}$ and beyond. Although each mMTC device occupies quite narrow bandwidth, the massive number of devices expected will generate a vast array of traffic and consume enormous spectrum resources. Therefore, it is necessary to proactively gather up and exploit fractional spectrum resources including guard bands that are secured but unused by the existing Long Term Evolution (LTE) systems. The guard band is originally secured as a margin for high out-of-band emission (OOBE) caused by the discontinuity between successive symbols in the cyclic prefix-based orthogonal frequency division multiplexing (CPOFDM), and new-waveforms enabling high OOBE suppression have been widely researched to efficiently allocate narrowband communication to the frequency gap. Time-domain windowing is a well-known signal processing technique for reducing OOBE with low complexity and a universal timedomain windowed OFDM (UTW-OFDM) with a long transition duration exceeding the CP length has demonstrated its ability in WLAN-based systems. In this paper, we apply UTW-OFDM to the LTE downlink system and comprehensively evaluate its performance under the channel models defined by 3GPP. Specifically, we evaluate OOBE reduction and block error rate (BLER) by computer simulation and clarify how far OOBE can be reduced without degrading communication quality. Furthermore, we estimate the implementation complexity of the proposed UTW-OFDM, the conventional CP-OFDM, and the universal filtered-OFDM (UF-OFDM) by calculating the number of required multiplications. These evaluation and estimation results demonstrate that the proposed UTW-OFDM is a practical new-waveform applicable to the $5 \mathrm{G}$ and beyond.

key words: CP-OFDM, UTW-OFDM, time-domain windowing, LTE, outof-band emission (OOBE), block error rate (BLER), complexity
\end{abstract}

\section{Introduction}

The fifth-generation mobile communications (5G) and beyond will support emerging all-new use cases collaborating with the Internet-of-things (IoT) and massive machine type communication (mMTC) services (e.g., monitoring systems with agricultural, structural, and industrial sensor devices, and telemedicine and e-healthcare systems with wearable devices) [1]. Although the required data rate of the IoT and mMTC devices is generally lower than that of the existing cellular devices, considerable spectrum resources will be consumed because an enormous number of devices simultaneously connect to the network. Millimeter waves being discussed as new frequency bands for the $5 \mathrm{G}$ and beyond can offer abundant spectrum resources, while the mMTC systems require a wide coverage at low cost and still use

\footnotetext{
Manuscript received August 23, 2018.

Manuscript revised January 1, 2019.

Manuscript publicized February 22, 2019.

$\dagger$ The authors are with Graduate School of Informatics, Kyoto University, Kyoto-shi, 606-8501 Japan.

a)E-mail: mizutani@i.kyoto-u.ac.jp

DOI: $10.1587 /$ transcom.2018EBP3242
}

the conventional microwave bands. In fact, new categories such as Long Term Evolution for machines (LTE-M) and narrow band IoT (NB-IoT), which have been standardized as conventional LTE-based low power wide area (LPWA) technologies, are supposed to use sub-GHz bands with narrow bandwidth (e.g., $1.4 \mathrm{MHz}$ for LTE-M and $200 \mathrm{kHz}$ for NBIoT [2]). In the emerging LTE-based LPWA and existing LTE systems, the cyclic prefix-based orthogonal frequency division multiplexing (CP-OFDM) is applied as a digital encoding scheme for downlink. In general, the CP-OFDM requires a guard band and/or an isolation distance as a margin because of its high out-of-band emission (OOBE) caused by the discontinuity between successive CP-OFDM symbols. Although the occupied bandwidth is quite narrow in the LTE-based LPWA systems, it is necessary to proactively gather up and exploit fractional spectrum resources including guard bands that are secured but unused. Since the conventional LTE and LTE-based LPWA systems are operated in the same microwave bands, new-waveforms with significant low OOBE have been widely researched to enhance spectral efficiency and to accommodate a massive number of mMTC devices with less margin.

New-waveforms with significant low OOBE are roughly categorized into two types according to applied schemes: filtering-based new-waveforms and windowing-based newwaveforms. The formers are generated by convoluting the transmission symbols with low pass filter coefficients in the time-domain to smooth the discontinuity at the beginning and the end of OFDM symbols, enabling OOBE reduction. The filter bank multicarrier (FBMC) [3], [4], the filtered OFDM (f-OFDM) [5], and the universal filtered OFDM (UFOFDM) [6]-[8] are typical but disparate new-waveforms based on filtering. In common, a filtering process to achieve sufficient OOBE reduction lavishes plenty of multiplications and increases size and complexity in the transmitter. Furthermore, an additional filtering process entails, in part, alterations of the conventional LTE transmission signal format. For example, the UF-OFDM applies zero-padding (ZP) insertion instead of CP insertion [7] and literally breaks compatibility with the conventional CP-OFDM. Such high complexity and low compatibility are major challenges for the smooth application of filtering-based new-waveforms to the $5 \mathrm{G}$ and beyond.

Time-domain windowing is a well-known signal processing technique for reducing OOBE with less complexity compared to the time-domain filtering. The windowing process can smooth the discontinuity between succes- 
sive CP-OFDM symbols by multiplying a windowing coefficient in the time-domain and thus, only one multiplication is consumed [9]-[11]. The OOBE reduction performance of the time-domain windowing depends on the physical layer (PHY) parameter set of the system (e.g., subcarrier interval, sampling frequency, symbol duration, CP length, etc.), an applied windowing function, and its transition duration (i.e., the number of time samples for smoothing). Here, long transition duration exceeding the $\mathrm{CP}$ length can reduce OOBE significantly but may deteriorate communication quality due to the inter-symbol interference (ISI) and inter-carrier interference (ICI) caused by intensive waveform shaping. Meanwhile, our previous works demonstrated that a channel coding scheme with error correction mitigated bit errors caused by the ISI and ICI and resulted in significant OOBE reduction without the degradation of communication quality [12]. We proposed the universal time-domain windowed OFDM (UTW-OFDM) in which the applied windowing function and its transition duration are selected appropriately and flexibly for the used PHY parameter set and channel condition in 2013 [13]. If necessary, a long transition duration exceeding the CP length can be applied in the proposed UTW-OFDM within a range not to affect the communication quality. In [12], the proposed UTW-OFDM was applied to the IEEE 802.11af system that is a WLAN system using the television white space (TVWS) [11]. For TVWS communication systems, leading countries regulated a strict spectrum mask to avoid interference with primary users (i.e., TV broadcasters). In our case, by applying a long transition duration exceeding the CP length, the transmission signal achieved the FCC requirement [14] while maintaining sufficient communication quality [12]. This result implies that the UTW-OFDM has the potential to reduce OOBE without degrading communication quality even in the LTE system since the turbo coding has a powerful error correction capability.

In this paper, we apply the UTW-OFDM to the LTE downlink system [15], [16] and comprehensively evaluate its performance. First, OOBE reduction performance of the UTW-OFDM-based LTE downlink transmission signal is evaluated by changing the UTW transition duration. Then, BLER characteristics are evaluated under the 3GPP channel models of the Extended Typical Urban (ETU) and the Extended Vehicular A (EVA) with a $70 \mathrm{~Hz}$ Doppler shift (i.e., ETU70 and EVA70) [17]. Furthermore, the implementation complexity of the UTW-OFDM is compared to the conventional CP-OFDM and the UF-OFDM. Our comprehensive evaluation results demonstrate the practicality of the UTWOFDM for the LTE-based 5G and beyond downlink.

The rest of this paper is organized as follows. The overviews of the conventional CP-OFDM and the OOBE reduced new-waveforms for the $5 \mathrm{G}$ and beyond are described in Sect. 2. Then, the transceiver architecture of the proposed UTW-OFDM-based LTE system is described in Sect. 3, and performance of the proposed UTW-OFDM-based LTE system is comprehensively evaluated in Sect. 4. In Sect. 5, we discuss the trade-off between OOBE reduction performance and BLER characteristics and evaluate implementation com- plexity to confirm the practicality. Finally, we conclude this paper in Sect. 6.

\section{Overview of New-Waveforms for OOBE Reduction}

In the existing fourth-generation mobile communications (4G), the CP-OFDM and the CP-DFTs-OFDM are applied as downlink and uplink radio access schemes, respectively. For the smooth transition from the $4 \mathrm{G}$ to the $5 \mathrm{G}$ and beyond, a CP-OFDM-based waveform will be ongoingly applied. Since the CP-OFDM has the drawback of high OOBE, various new-waveforms with low OOBE have been proposed. As shown in Fig. 1, the new-waveforms can be roughly categorized into two types: filtering-based new-waveforms [3]-[8] and windowing-based new-waveforms [12], [13], [18]). In the following subsections, the conventional CP-OFDM and various new-waveforms with low OOBE are overviewed.

\subsection{Conventional CP-OFDM}

The conventional CP-OFDM is a multi-carrier waveform widely used for the existing wireless communication systems. Let us define the $n$-th subcarrier signal of the $m$-th symbol as $x_{m, n}$, then the CP-OFDM waveform for the $m$-th symbol $\boldsymbol{s}_{m}^{\mathrm{CP}} \in \mathbb{C}^{N+N_{\mathrm{G}}}$ can be generated as follows:

$$
\begin{aligned}
& \boldsymbol{s}_{m}^{\mathrm{CP}}=\boldsymbol{F}_{\mathrm{G}}^{-1} \boldsymbol{x}_{m}, \\
& \boldsymbol{x}_{m}=\left[x_{m, 0}, x_{m, 1}, \cdots, x_{m, N-1}\right]^{\mathrm{T}}, \\
& \left(\boldsymbol{F}_{\mathrm{G}}^{-1}\right)_{(g, q)}=\exp \left[\frac{\mathrm{j} 2 \pi q\left(g-N_{\mathrm{G}}\right)}{N}\right],
\end{aligned}
$$

where $N$ and $N_{\mathrm{G}}$ denote the number of subcarriers in one symbol and the number of samples of the $\mathrm{CP}$, respectively, and $\boldsymbol{F}_{\mathrm{G}}^{-1} \in \mathbb{C}^{N+N_{\mathrm{G}} \times N}$ is an inverse discrete Fourier transform (IDFT) matrix including the CP insertion with $0 \leq g<N+N_{\mathrm{G}}$ and $0 \leq q<N$. Each OFDM symbol with a $\mathrm{CP}$ has the property of a continuous signal, while the $\mathrm{CP}$ OFDM transmission signal has discontinuity at the transition point between successive symbols, causing high OOBE. Since higher OOBE limits the effective use of the spectrum at neighboring channels, a new-waveform with low-OOBE is required for the $5 \mathrm{G}$ and beyond.

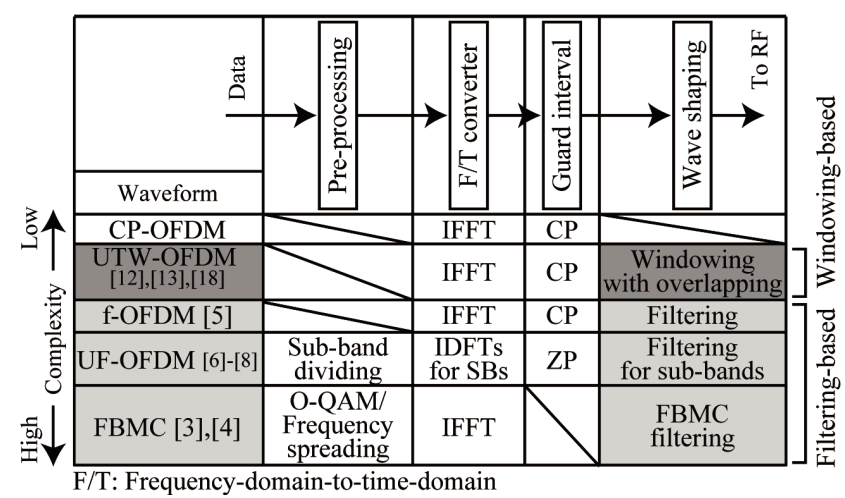

Fig. 1 New-waveforms with low OOBE for $5 \mathrm{G}$ and beyond. 


\subsection{Filtering-Based New-Waveforms}

The FBMC [3], [4], the f-OFDM [5], and the UF-OFDM [6][8] are typical filtering-based new-waveforms. In particular, the FBMC offers tremendous OOBE reduction potential as it uses FBMC filtering for wave shaping (e.g., the OOBE of the FBMC with LTE parameters is lower than $-130 \mathrm{dBr}$ ( $\mathrm{dB}$ relative to the maximum power spectrum density (PSD) of the transmit signal) at the channel-edge [4]). However, FBMC-based systems have poor compatibility with the conventional LTE system because the FBMC requires some alterations of the LTE parameters and transceiver architecture. For instance, to apply the FBMC filtering, offset-quadrature amplitude modulation (O-QAM) and a frequency spreading process are additionally required in the pre-processing as shown in Fig. 1.

On the other hand, the f-OFDM is more compatible with the conventional CP-OFDM than the FBMC because only its transmitter needs to be changed with the addition of time-domain filtering for wave shaping. Unfortunately, the filtering process uses a large number of taps for f-OFDM and implementation is hindered by the huge number of multiplications needed. Moreover, the f-OFDM requires different filter designs depending on the number of transmission subcarriers.

In the UF-OFDM, subcarriers in the transmission band are grouped into several sub-bands (e.g., 300 subcarriers are divided into 25 sub-bands and each sub-band has 12 subcarriers when the $5 \mathrm{MHz}$ bandwidth LTE downlink parameters are applied [7]) and OFDM symbol for each sub-band is generated by using IDFT. Then, the universal filtering is applied to each sub-band OFDM symbol before generating the UFOFDM symbol. In the UF-OFDM, the same filter design can be applied to each sub-band OFDM symbol unlike the f-OFDM. When the $5 \mathrm{MHz}$ bandwidth LTE downlink parameters are applied, the UF-OFDM can reduce the OOBE at the channel edge (i.e., an offset frequency of $2.5 \mathrm{MHz}$ ) by about $25 \mathrm{~dB}$ compared with the conventional CP-OFDM. However, the UF-OFDM also requires some alterations of the conventional LTE parameters. For example, ZP insertion is required instead of $\mathrm{CP}$ insertion as shown in Fig. 1 because the impulse response of the universal filter is used as the guard interval. Furthermore, the filtering process consumes a large number of multiplications and thus, increases implementation complexity.

\subsection{Windowing-Based New-Waveforms}

\subsubsection{Time-Domain Windowing for OOBE Suppression}

Time-domain windowing is a well-known signal processing technique for reducing OOBE of the CP-OFDM with low complexity [9]-[11]. To reduce OOBE, the discontinuity between successive CP-OFDM symbols is smoothed by multiplying a windowing coefficient in the time-domain with only one multiplication. Here, the both edges of the windowed CP-OFDM symbol can be overlapped with the successive windowed CP-OFDM symbols for retaining constant transmission power. The OOBE suppression performance depends on PHY parameters of the applied system, an applied windowing function, and its transition duration. In general, longer transition duration can reduce OOBE more significantly but deteriorates communication quality due to the ISI and ICI caused by waveform distortion, especially in the case that the transition duration exceeds the CP length.

This scheme is currently applied to the existing CPOFDM-based wireless communication systems such as the IEEE $802.11 \mathrm{a} / \mathrm{g} / \mathrm{n} / \mathrm{ac}$. Spectrum masks defined in these systems are not strict and thus, the minimum transition duration (i.e., one or few samples in the time-domain) is sufficient for achieving the requirements. In existing WLAN system with a sampling frequency of $20 \mathrm{MHz}$ [11], for example, the amplitude of the transmission signal at both edges of the CP-OFDM symbol is reduced to half, and the leftmost sample of the $m$-th CP-OFDM symbol and the rightmost one of the $(m-1)$-th CP-OFDM are overlapped. By applying this scheme, the transmission waveform satisfies the spectrum mask requirement defined as $0 \mathrm{dBr},-20 \mathrm{dBr},-28 \mathrm{dBr}$, and $-40 \mathrm{dBr}$ at an offset frequency of $9 \mathrm{MHz}, 11 \mathrm{MHz}, 20 \mathrm{MHz}$, and $30 \mathrm{MHz}$, respectively, with a $100 \mathrm{kHz}$ resolution bandwidth (RBW) [11].

\subsubsection{UTW-OFDM for TVWS Communication System}

In the case of the IEEE 802.11af [11], the time-domain windowing exerted its full potential to achieve a strict spectrum requirement. The IEEE 802.11af was standardized as the WLAN system operating in the TV white-spaces (TVWS). To protect the primary users (i.e., TV broadcasting systems), leading countries imposed strict transmission spectrum mask on the TVWS communication systems during the operation. For example, the FCC in the U.S. limits in-band PSD as $+2.6 \mathrm{dBm} / 100 \mathrm{kHz}$ and OOBE as $-52.8 \mathrm{dBm} / 100 \mathrm{kHz}$ (i.e., $-55.4 \mathrm{dBr} / 100 \mathrm{kHz}$ ) in the entire $\mathrm{TV}$ band even at the channel-edge with an offset frequency of $3 \mathrm{MHz}$ for mobile devices (defined as Mode II TV band devices in [14]). Since the transmission signal must achieve this requirement, it is necessary to consider a more severe spectrum mask since the distortion caused by the nonlinearity of the RF circuit might deteriorate OOBE.

The implementation of an array of analog RF filters specified for each channel is one of the simplest methods to comply with the strict transmission mask, while the implementation of a large number of not small analog RF filters (e.g., for the TV band in the UHF, 40 channels are assigned from $470 \mathrm{MHz}$ to $710 \mathrm{MHz}$ in Japan, and 56 channels are assigned from $470 \mathrm{MHz}$ to $806 \mathrm{MHz}$ in the U.S.) increases size and cost and impairs the design flexibility. A digital baseband filter is one of the options to relax the requirements for RF filters and can offer flexible operation by changing its parameters according to the using channel. Meanwhile, more than a hundred taps are required to comply with the FCC regulation [19]. 
The UTW-OFDM with a long transition duration exceeding the CP length achieved the strict spectrum mask requirement and demonstrated its practicality for the IEEE 802.11af [12]. Concerned negative effects of the ICI and ISI were greatly alleviated by the convolutional coding (i.e., forward error correction (FEC)). In the case of 64QAM and a coding rate of $5 / 6$ with a $6 \mathrm{MHz}$ bandwidth and a $\mathrm{CP}$ length of $1 / 4$, the UTW-OFDM with the raised-cosine window achieved an averaged PSD of about $-95 \mathrm{dBr}$ (i.e., around $40 \mathrm{~dB}$ margin for the FCC regulation) at the channel-edge without throughput degradation under the spatial channel model extended (SCME) micro urban channel model [20]. This result indicates that the UTW-OFDM with a long transition duration exceeding the $\mathrm{CP}$ length has the potential to greatly reduce OOBE without degrading communication quality even in the LTE system since the turbo coding has a powerful error correction capability.

\section{Transceiver Architecture for UTW-OFDM-Based LTE Downlink System}

Fundamental PHY parameters such as a sampling frequency, a subcarrier spacing, a CP length, and a channel coding scheme are different between the IEEE 802.11 af and the LTE. In this section, we explain how to apply the UTW-OFDM to the LTE downlink system with a proposed transceiver architecture.

\subsection{Transmitter Architecture}

Figure 2 shows the proposed UTW-OFDM transmitter architecture. The transmission binary data are channel-coded, modulated, and then, allocated to appropriate subcarriers by the PHY layer mapping controller (e.g., the PHY scheduler). The allocated subcarrier signals are converted to the time-domain OFDM symbol by the IDFT or the inverse fast Fourier transform (IFFT). Let us define the sampling frequency and the length of the time-domain OFDM symbol as $F_{\mathrm{S}}$ and $T_{\mathrm{F}}$, respectively, then the number of time-domain samples of the OFDM symbol can be expressed as $N=T_{\mathrm{F}} F_{\mathrm{S}}$. After that, the $\mathrm{CP}$ for reducing the effect of multi-path propagation delay and overlap margins (OMs) are generated by copying the beginning and the end of the OFDM symbol as shown in Fig. 3(a). Since the OFDM symbol has periodicity, the symbol with inserted $\mathrm{CP}$ and $\mathrm{OMs}$ retains continuity.

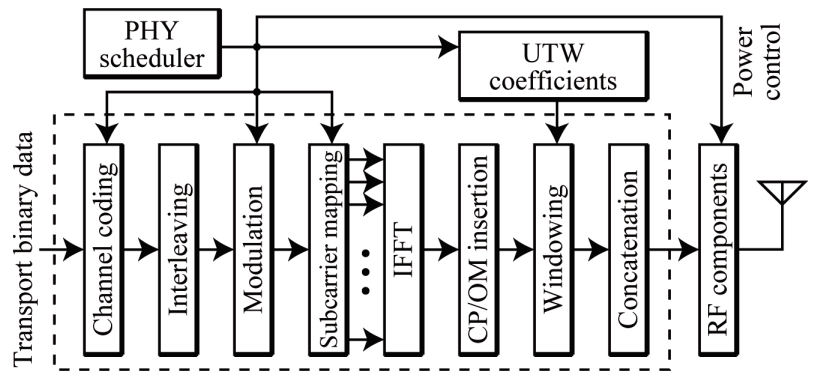

Fig. 2 Block diagram of transmitter for proposed UTW-OFDM.
The $m$-th OFDM symbol $\boldsymbol{s}_{m} \in \mathbb{C}^{N_{\mathrm{GM}}}$ with the $N_{\mathrm{G}}$ samples $\mathrm{CP}$, the $N_{\mathrm{M}}$ samples prefix-OM, and the $\left(N_{\mathrm{M}}+1\right)$ samples suffix-OM can be expressed as

$$
s_{m}=F_{\mathrm{GM}}^{-1} \boldsymbol{x}_{m},
$$

where an IDFT matrix including the inserted $\mathrm{CP}$ and $\mathrm{OMs}$ $\boldsymbol{F}_{\mathrm{GM}}^{-1} \in \mathbb{C}^{N_{\mathrm{GM}} \times N}$ is defined by

$$
\left(\boldsymbol{F}_{\mathrm{GM}}^{-1}\right)_{(p, q)}=\exp \left[\frac{\mathrm{j} 2 \pi q\left(p-N_{\mathrm{G}}-N_{\mathrm{M}}\right)}{N}\right],
$$

where $0 \leq p<N_{\mathrm{GM}}$ and $N_{\mathrm{GM}}=N+N_{\mathrm{G}}+2 N_{\mathrm{M}}+1$. By multiplexing a UTW to the OFDM symbol with the CP and OMs insertion as shown in Fig. 3(b), the $m$-th UTW-OFDM symbol $\boldsymbol{s}_{m}^{\mathrm{UTW}} \in \mathbb{C}^{N_{\mathrm{GM}}}$ can be generated as

$$
\boldsymbol{s}_{m}^{\mathrm{UTW}}=\boldsymbol{W}^{\mathrm{UTW}} \boldsymbol{F}_{\mathrm{GM}}^{-1} \boldsymbol{x}_{m},
$$

where $\boldsymbol{W}^{\mathrm{UTW}}=\operatorname{diag}\left(\boldsymbol{w}^{\mathrm{UTW}}\right) \in \mathbb{C}^{N_{\mathrm{GM}} \times N_{\mathrm{GM}}}$ denotes the UTW matrix and $\operatorname{diag}(\boldsymbol{d})$ represents a diagonal matrix generation function by using a vertical vector $\boldsymbol{d}$. Here, $\boldsymbol{w}^{\mathrm{UTW}} \in \mathbb{C}^{N_{\mathrm{GM}}}$ is a UTW vector and can be expressed as follows:

$$
\boldsymbol{w}^{\mathrm{UTW}}=\left[\begin{array}{c}
\mathbf{0}^{\left(\mathrm{N}_{\mathrm{M}}-N_{\mathrm{TR}} / 2\right) \times 1} \\
\boldsymbol{w}_{\mathrm{TR}} \\
\mathbf{1}^{\left(N+N_{\mathrm{G}}-N_{\mathrm{TR}}+1\right) \times 1} \\
\boldsymbol{w}_{\mathrm{TR}}^{\mathrm{I}} \\
\mathbf{0}^{\left(N_{\mathrm{M}}-N_{\mathrm{TR}} / 2\right) \times 1}
\end{array}\right],
$$

where $\mathbf{0}^{P \times Q}$ is a $P$-by- $Q$ zero matrix and $\mathbf{1}^{P \times Q}$ is a $P$-by$Q$ matrix in which all factors are one. Here, $N_{\mathrm{TR}}$ denotes the UTW transition duration, in which the UTW amplitude changes from zero to one or vice versa. A UTW transition vector $\boldsymbol{w}_{\mathrm{TR}} \in \mathbb{C}^{N_{\mathrm{TR}}}$ and an inverse UTW transition vector $\boldsymbol{w}_{\mathrm{TR}}^{\mathrm{I}} \in \mathbb{C}^{N_{\mathrm{TR}}}$ are defined by

$$
\begin{aligned}
& \boldsymbol{w}_{\mathrm{TR}}=\left[w_{0}, w_{1}, \cdots, w_{N_{\mathrm{TR}}-2}, w_{N_{\mathrm{TR}}-1}\right]^{\mathrm{T}}, \\
& \boldsymbol{w}_{\mathrm{TR}}^{\mathrm{I}}=\left[w_{N_{\mathrm{TR}}-1}, w_{N_{\mathrm{TR}}-2}, \cdots, w_{1}, w_{0}\right]^{\mathrm{T}} .
\end{aligned}
$$

A variety of windowing functions can be applied as the UTW transition vectors. In the case of the raised-cosine windowing function, $w_{n}$ can be defined by

$$
w_{n}=0.5-0.5 \cos \left(\frac{\pi n}{N_{\mathrm{TR}}}\right) .
$$

Finally, each UTW-OFDM symbol is concatenated with the successive symbols by overlapping the OM durations to generate the UTW-OFDM transmission signal as shown in Fig. 3(c). In the UTW-OFDM, applied windowing function and its transition duration are flexibly configured according to the system parameters including the RF and the baseband circuit characteristics of the transmitter, desired transmission power, and a propagation environment [13].

\subsection{Receiver Architecture}

The receiver architecture of the conventional CP-OFDM 


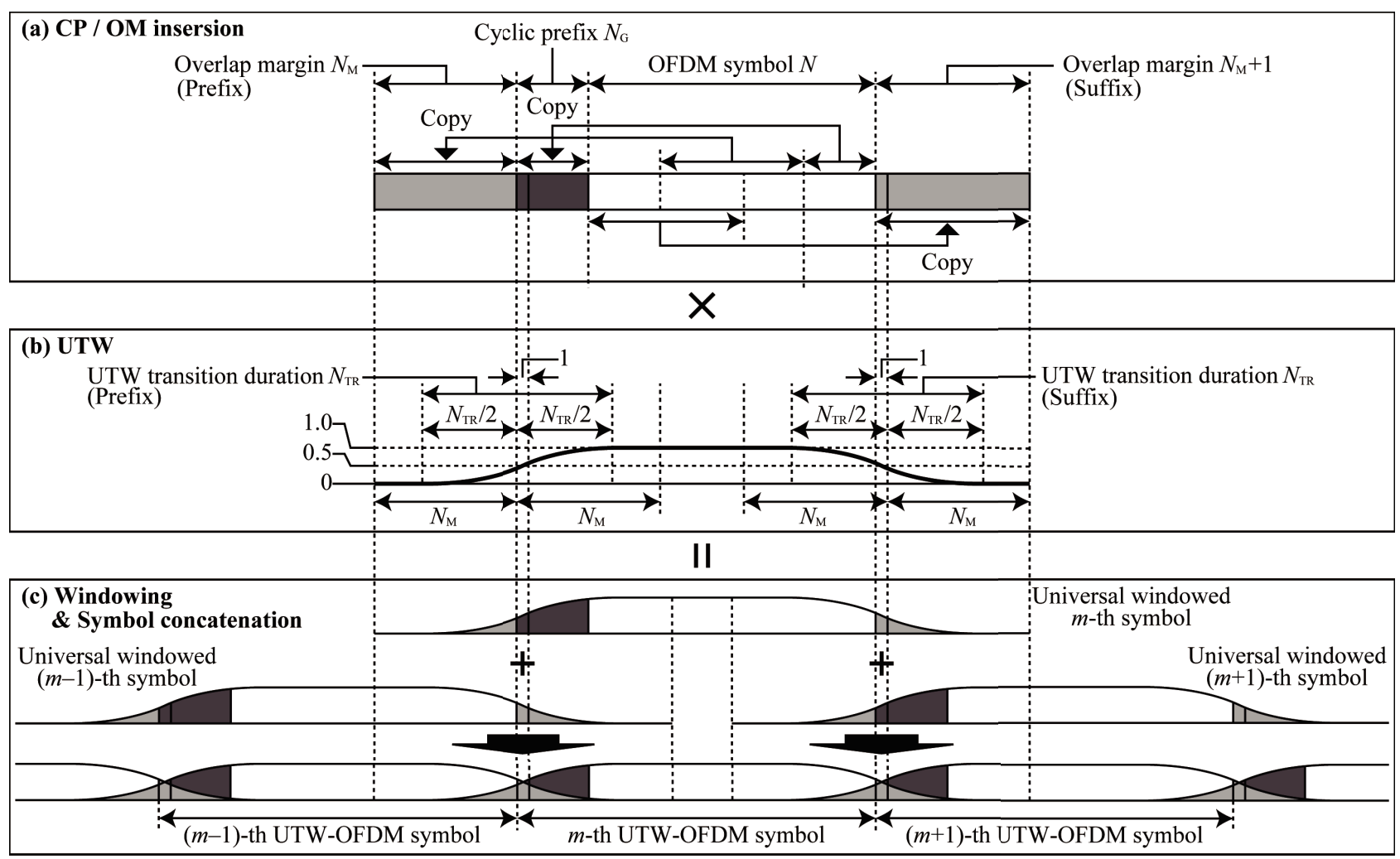

Fig.3 Transmission signal generation flow of proposed UTW-OFDM.

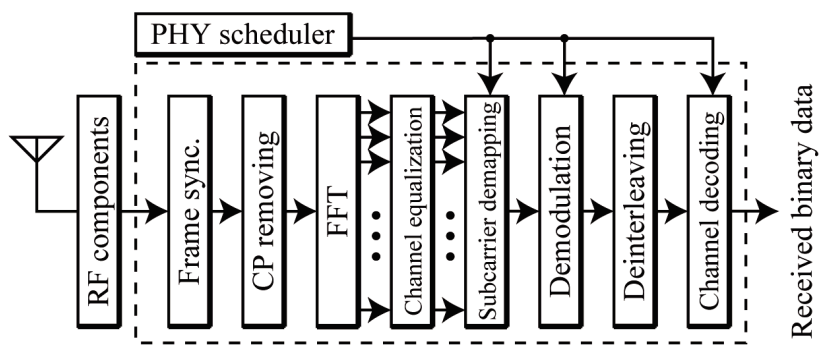

Fig. 4 Block diagram of receiver for proposed UTW-OFDM.

shown in Fig. 4 can be used for the proposed UTW-OFDM without any modification. Let us define the propagation channel matrix as $\boldsymbol{H}_{m} \in \mathbb{C}^{N_{\mathrm{GM}} \times N_{\mathrm{GM}}}$ and the Gaussian noise with zero mean and $\sigma^{2}$ variance for the $n$-th subcarrier of the $m$-th symbol as $n_{m, n}$, then the $m$-th symbol duration of the UTW-OFDM received signal $\boldsymbol{r}_{m} \in \mathbb{C}^{N_{\mathrm{GM}}}$ can be expressed as follows:

$$
\begin{aligned}
& \boldsymbol{r}_{m}=\boldsymbol{H}_{m} \boldsymbol{s}_{m}^{\mathrm{UTW}, \dagger}+\boldsymbol{n}_{m}, \\
& \boldsymbol{n}_{m}=\left[n_{m,-\left(N_{\mathrm{M}}+N_{\mathrm{G}}\right)}, \cdots, n_{m, 0}, \cdots, n_{m, N+N_{\mathrm{M}}}\right]^{\mathrm{T}},
\end{aligned}
$$

where $\boldsymbol{s}_{m}^{\mathrm{UTW}, \dagger} \in \mathbb{C}^{N_{\mathrm{GM}}}$ denotes the ISI component for the $m$-th UTW-OFDM symbol from the successive symbols and can be described as follows:

$$
\boldsymbol{s}_{m}^{\mathrm{UTW}, \dagger}=\boldsymbol{s}_{m}^{\mathrm{UTW}}+\boldsymbol{s}_{m}^{(-)}+\boldsymbol{s}_{m}^{(+)},
$$

where $\boldsymbol{s}_{m}^{(-)} \in \mathbb{C}^{N_{\mathrm{GM}}}$ and $\boldsymbol{s}_{m}^{(+)} \in \mathbb{C}^{N_{\mathrm{GM}}}$ represent the ISI components from the $(m-1)$-th symbol and the $(m+1)$-th symbol, respectively, and can be calculated by

$$
\begin{aligned}
\boldsymbol{s}_{m}^{(-)} & =\boldsymbol{W}_{(-)}^{\mathrm{UTW}}\left[\left(\boldsymbol{F}_{(-)}^{-1}\right)^{\mathrm{T}}, \mathbf{0}^{N \times\left(N+N_{\mathrm{G}}\right)}\right]^{\mathrm{T}} \boldsymbol{x}_{m-1}, \\
\boldsymbol{s}_{m}^{(+)} & =\boldsymbol{W}_{(+)}^{\mathrm{UTW}}\left[\mathbf{0}^{N \times\left(N+N_{\mathrm{G}}\right)},\left(\boldsymbol{F}_{(+)}^{-1}\right)^{\mathrm{T}}\right]^{\mathrm{T}} \boldsymbol{x}_{m+1},
\end{aligned}
$$

where $\boldsymbol{F}_{(-)}^{-1}$ and $\boldsymbol{F}_{(+)}^{-1}$ denote IDFT matrices to express ISI components from the $(m-1)$-th symbol and the $(m+1)$-th symbol, respectively, and these ISI components are defined as follows:

$$
\begin{aligned}
& \left(\boldsymbol{F}_{(-)}^{-1}\right)_{\left(p_{(-)}, q\right)}=\exp \left[\frac{\mathrm{j} 2 \pi q\left(p_{(-)}-N_{\mathrm{G}}-N_{\mathrm{M}}\right)}{N}\right], \\
& \left(\boldsymbol{F}_{(+)}^{-1}\right)_{\left(p_{(+)}, q\right)}=\exp \left[\frac{\mathrm{j} 2 \pi q\left(p_{(+)}-N_{\mathrm{G}}-N_{\mathrm{M}}\right)}{N}\right],
\end{aligned}
$$

where $N+N_{\mathrm{G}}<p_{(-)}<N_{\mathrm{GM}}$ and $0 \leq p_{(+)} \leq 2 N_{\mathrm{M}}+1$. Here, $\boldsymbol{W}_{(-)}^{\mathrm{UTW}}=\operatorname{diag}\left(\boldsymbol{w}_{(-)}^{\mathrm{UTW}}\right) \in \mathbb{C}^{N_{\mathrm{GM}} \times N_{\mathrm{GM}}}$ and $\boldsymbol{W}_{(+)}^{\mathrm{UTW}}=$ $\operatorname{diag}\left(\boldsymbol{w}_{(+)}^{\mathrm{UTW}}\right) \in \mathbb{C}^{N_{\mathrm{GM}} \times N_{\mathrm{GM}}}$ representing the UTW of the $(m-1)$-th and $(m+1)$-th symbols, respectively, can be expressed as

$$
\boldsymbol{w}_{(-)}^{\mathrm{UTW}}=\left[\begin{array}{c}
\mathbf{1}^{\left(N_{\mathrm{M}}-\frac{N_{\mathrm{TR}}}{2}\right) \times 1} \\
\boldsymbol{w}_{\mathrm{TR}}^{\mathrm{I}} \\
\mathbf{0}^{\left(N+N_{\mathrm{G}}+N_{\mathrm{M}}-\frac{N_{\mathrm{TR}}}{2}+1\right) \times 1}
\end{array}\right],
$$




$$
\boldsymbol{w}_{(+)}^{\mathrm{UTW}}=\left[\begin{array}{c}
\boldsymbol{0}^{\left(N+N_{\mathrm{G}}+N_{\mathrm{M}}-\frac{N_{\mathrm{TR}}}{2}+1\right) \times 1} \\
\boldsymbol{w}_{\mathrm{TR}}^{\mathrm{I}} \\
\mathbf{1}^{\left(N_{\mathrm{M}}-\frac{N_{\mathrm{TR}}}{2}\right) \times 1}
\end{array}\right] .
$$

The subcarrier signal vector of the $m$-th symbol $\boldsymbol{y}_{m} \in \mathbb{C}^{N}$ is obtained by the frame synchronization, the $\mathrm{CP}$ removal, and the fast Fourier transform (FFT) operation as follows:

$$
\boldsymbol{y}_{m}=\boldsymbol{F D} \boldsymbol{r}_{m},
$$

where $\boldsymbol{F} \in \mathbb{C}^{N \times N}$ and $\boldsymbol{D} \in \mathbb{C}^{N_{\mathrm{GM}} \times N}$ are an FFT matrix and a frame synchronization matrix, respectively, and defined by

$$
\begin{aligned}
& (\boldsymbol{F})_{(r, q)}=\exp \left[\frac{-\mathrm{j} 2 \pi q r}{N}\right], \\
& \boldsymbol{D}=\left[\boldsymbol{0}^{\left(N_{\mathrm{GM}} \times N_{\mathrm{S}}\right)}, \boldsymbol{I}^{(N)}, \boldsymbol{0}^{\left(N_{\mathrm{GM}} \times\left(N_{\mathrm{GM}}-N_{\mathrm{S}}-N\right)\right)}\right],
\end{aligned}
$$

where $0 \leq r<N, \boldsymbol{I}^{(N)} \in \mathbb{C}^{N \times N}$ denotes the $N$-by- $N$ identity matrix, and $N_{\mathrm{S}}$ represents a frame synchronization offset. After that, channel estimation and equalization are applied to each subcarrier signal by using reference signals (RSs). Finally, demodulation and channel decoding are applied to obtain received binary data.

\section{Performance Evaluation}

In this section, we evaluate the performance of the proposed UTW-OFDM-based LTE downlink system [15], [16] by computer simulation from the aspects of OOBE reduction and BLER.

\subsection{OOBE Reduction}

The OOBE reduction performance of the proposed UTWOFDM is evaluated with the $5 \mathrm{MHz}$ bandwidth LTE downlink parameters shown in Table 1 [21]. Figure 5 shows the average PSD of the proposed UTW-OFDM with an RBW of $100 \mathrm{kHz}$ and an oversampling factor of four. In this evaluation, the raised-cosine windowing function expressed in Eq. (10) is applied as the UTW. Obviously, the proposed UTW-OFDM remarkably reduces OOBE compared to the conventional CP-OFDM by increasing the UTW transition duration $N_{\mathrm{TR}}$. For the quantitative evaluation of the OOBE reduction, the averaged PSD at the channel-edge is plotted as a function of relative $N_{\mathrm{TR}}$ as shown in Fig. 6. From the result, the proposed UTWOFDM consistently reduces OOBE regardless of the modulation scheme. The averaged PSD of the conventional CPOFDM at the channel-edge is $-26 \mathrm{dBr} / 100 \mathrm{kHz}$. By applying time-domain window with minimum transition duration (i.e., $N_{\mathrm{TR}} / N=2 / 512=0.004$ ), the averaged PSD of the $\mathrm{CP}-\mathrm{OFDM}$ at an offset frequency of $5 \mathrm{MHz}$ is reduced by about $10 \mathrm{~dB}$, while the averaged PSD at the channel-edge is same. In contrast, the averaged PSD of the proposed UTW-OFDM-based signal at the channel-edge reduces to $-44 \mathrm{dBr} / 100 \mathrm{kHz}$, when the relative UTW transition duration $N_{\mathrm{TR}} / N$ is set to $36 / 512 \approx 0.07$ equivalent to the
Table 1 Specification of evaluated LTE downlink signal [21].

\begin{tabular}{ll}
\hline Parameters & Value \\
\hline Signal format & LTE downlink (Rel. 8.0) \\
Air interface & UTW-OFDM \\
Channel bandwidth & $5.0 \mathrm{MHz}$ \\
Sampling frequency & $7.68 \mathrm{MHz}$ \\
FFT size $N$ & 512 \\
CP rate (1st and 8th symbols) & $40 / 512$ \\
CP rate (2nd-7th and 9th-14th symbols) & $36 / 512$ \\
Number of subcarrier per one OFDM symbol & 300 \\
Number of resource blocks per one slot & 25 \\
Number of slots per one sub-frame & 2 \\
Occupied bandwidth & $4.5 \mathrm{MHz}$ \\
Guard band & $0.25 \mathrm{MHz}$ on both edges \\
\hline
\end{tabular}

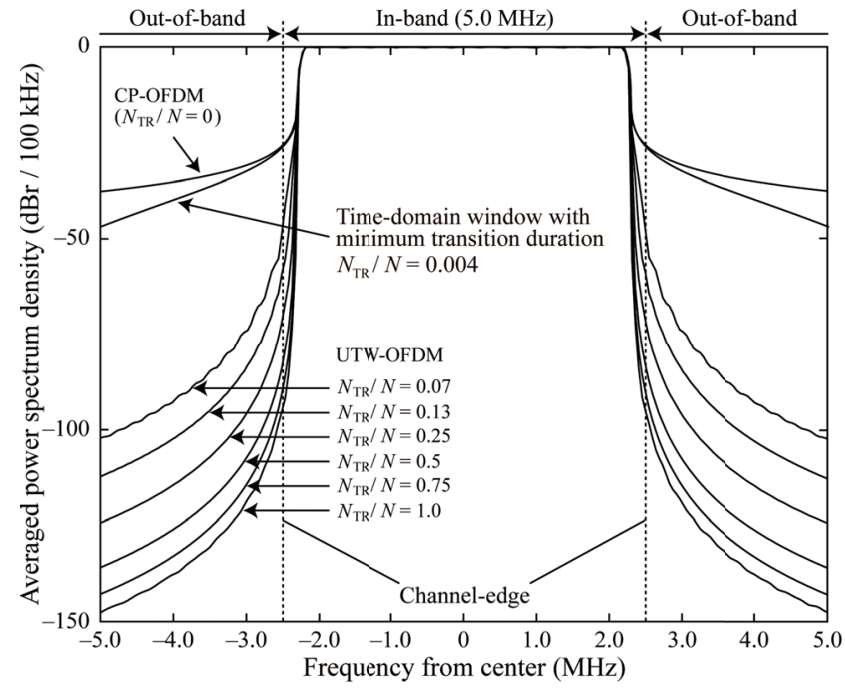

Fig. 5 PSD of proposed UTW-OFDM-based LTE downlink signal.

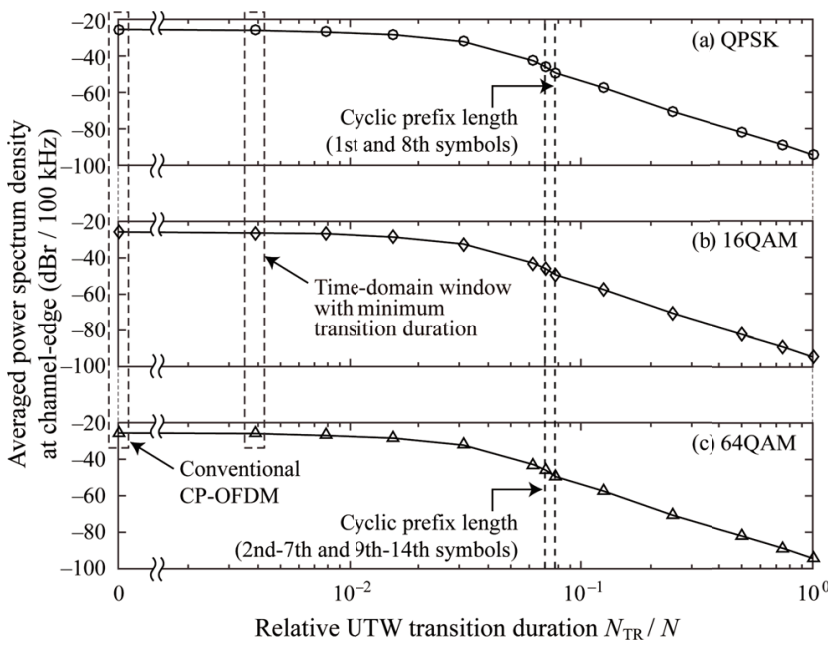

Fig. 6 OOBE reduction performance of proposed UTW-OFDM-based LTE downlink signal at channel-edge.

Normal-CP length $(4.7 \mu \mathrm{s})$. Moreover, when $N_{\mathrm{TR}} / N$ is set to $128 / 512=0.25,256 / 512=0.5$, and $512 / 512=1.0$, the averaged PSD at the channel-edge becomes $-71,-82$, and $-94 \mathrm{dBr} / 100 \mathrm{kHz}$ (i.e., reduced by 45,56 , and $68 \mathrm{~dB}$ compared to the conventional CP-OFDM-based signal), respectively. 
Table 2 Parameters for BLER evaluation.

\begin{tabular}{|c|c|c|}
\hline Parameters & \multicolumn{2}{|l|}{ Value } \\
\hline Transmission parameters & \multicolumn{2}{|c|}{ LTE-OFDM (see Table 1) } \\
\hline Modulation and coding rate $R$ & \multicolumn{2}{|c|}{ QPSK, $R=0.50$ (MCS 9) } \\
\hline & \multicolumn{2}{|c|}{ 16QAM, $R=0.48(\mathrm{MCS} 16)$} \\
\hline & \multicolumn{2}{|c|}{ 64QAM, $R=0.48(\mathrm{MCS} 22)$} \\
\hline & \multicolumn{2}{|c|}{ 64QAM, $R=0.57(\mathrm{MCS} 24)$} \\
\hline & \multicolumn{2}{|c|}{ 64QAM, $R=0.64(\mathrm{MCS} 26)$} \\
\hline & \multicolumn{2}{|c|}{ 64QAM, $R=0.66(\mathrm{MCS} 27)$} \\
\hline & \multicolumn{2}{|c|}{ 64QAM, $R=0.77(\mathrm{MCS} 28)$} \\
\hline Channel coding scheme & \multicolumn{2}{|c|}{ Turbo coding } \\
\hline Decoding algorithm & \multicolumn{2}{|c|}{ Max-Log MAP } \\
\hline $\begin{array}{l}\text { Number of turbo decoding } \\
\text { iteration }\end{array}$ & \multicolumn{2}{|c|}{5} \\
\hline UTW function & \multicolumn{2}{|c|}{ Raised-cosine window } \\
\hline Center frequency & \multicolumn{2}{|c|}{$2.5 \mathrm{GHz}$} \\
\hline \multirow[t]{2}{*}{ Channel model } & EVA70 & $\begin{array}{l}\text { Extended Vehicular A model } \\
\text { with } 70 \mathrm{~Hz} \text { Doppler shift } \\
(30.2 \mathrm{~km} / \mathrm{h} \text { at } 2.5 \mathrm{GHz})\end{array}$ \\
\hline & ETU70 & $\begin{array}{l}\text { Extended Typical Urban } \\
\text { model with } 70 \mathrm{~Hz} \text { Doppler } \\
\text { shift }(30.2 \mathrm{~km} / \mathrm{h} \text { at } 2.5 \mathrm{GHz})\end{array}$ \\
\hline
\end{tabular}

Table 3 3GPP channel models [18].

\begin{tabular}{ll|ll}
\hline Extended Vehicular A model (EVA) & $\begin{array}{l}\text { Extended Typical Urban model } \\
(\text { ETU) }\end{array}$ & $\begin{array}{l}\text { Excess tap delay } \\
(\mathrm{ns})\end{array}$ & $\begin{array}{l}\text { Relative power } \\
(\mathrm{dB})\end{array}$ \\
\hline $\begin{array}{l}\text { Excess tap delay } \\
(\mathrm{ns})\end{array}$ & $\begin{array}{l}\text { Relative power } \\
(\mathrm{dB})\end{array}$ & 0 & -1.0 \\
\hline 0 & 0 & 50 & -1.0 \\
30 & -1.5 & 120 & -1.0 \\
150 & -1.4 & 200 & 0 \\
310 & -3.6 & 230 & 0 \\
370 & -0.6 & 500 & 0 \\
710 & -9.1 & 1,600 & -3.0 \\
1,090 & -7.0 & 2,300 & -5.0 \\
1,730 & -12.0 & 5,000 & -7.0 \\
2,510 & -16.9 & & \\
\hline
\end{tabular}

\subsection{Block Error Rate (BLER)}

BLER characteristics of the proposed UTW-OFDM are evaluated by computer simulation with the $5 \mathrm{MHz}$ bandwidth LTE downlink parameters in Table 1. Table 2 shows the simulation parameters. Because the proposed UTW-OFDM eats away the effective CP length by applying the long UTW transition duration, two kinds of channel models assuming different multipath fading environments are considered to confirm the effect of the long-delay multipath fading; the 3GPP EVA channel model with a $2.5 \mu$ s maximum delay and the 3GPP ETU channel model with a $5.0 \mu$ s maximum delay summarized in Table 3 [17]. Doppler shift is set to $70 \mathrm{~Hz}$ (i.e., $30.2 \mathrm{~km} / \mathrm{h}$ at a carrier frequency of $2.5 \mathrm{GHz}$ ). For the evaluations, BLER $=10^{-1}$ (i.e., the target BLER for channel quality indicator (CQI) table of LTE [22]-[24]) and $\mathrm{BLER}=10^{-3}$ (i.e., the reference BLER to achieve an almost $100 \%$ throughput without hybrid automatic repeat request (HARQ) [25], [26]) are used as the target BLER. In the reception process, the channels at the RSs, which are known signals at both transmitter and receiver, are first estimated, and then, the channels of the resource elements (REs) are estimated by interpolation and extrapolation in the time-domain as shown in Fig. 7(a). Secondly, the channels of the remaining REs are estimated by interpolation and extrapolation in the frequency-domain as shown in Fig. 7(b).

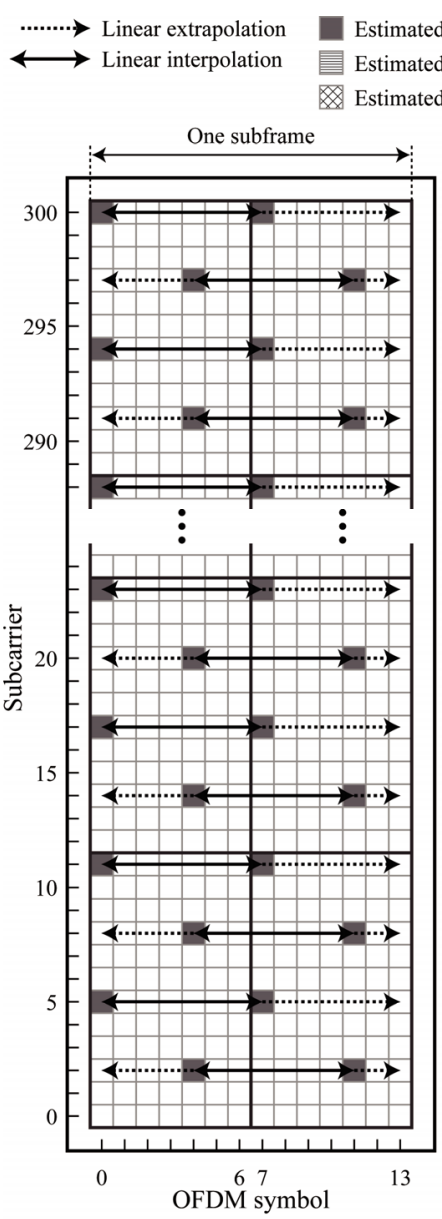

(a) 1st step stimated CSI by Reference signal (RS)

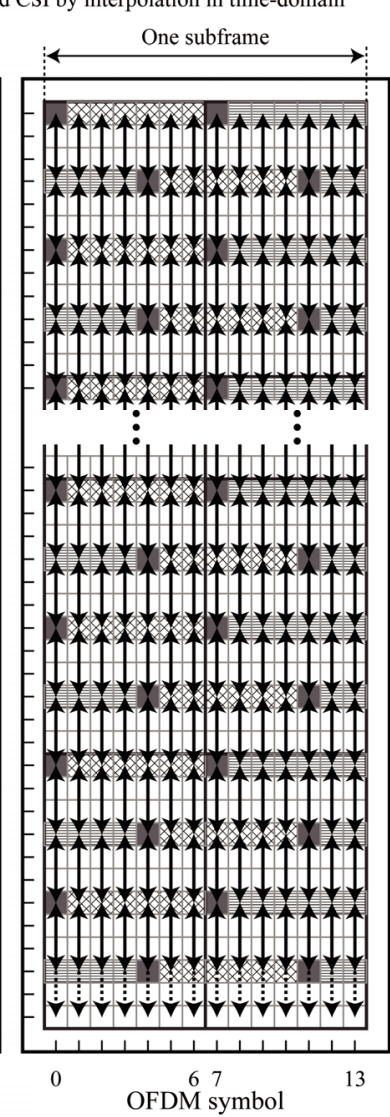

(b) 2nd step
CSI by extrapolation in time-domain

Fig. 7 Channel estimation process.

\subsubsection{Evaluation under EVA70 Channel Model}

Figure 8 shows the BLER characteristics of the proposed UTW-OFDM-based LTE downlink system under the 3GPP EVA70 channel model with a variety of modulation and coding schemes (MCSs): MCS-9 (i.e., QPSK with coding rate $R=0.50$ ), MCS-16 (i.e., 16QAM with $R=0.48$ ), MCS22 (i.e., 64QAM with $R=0.48$ ), MCS-24 (i.e., 64QAM with $R=0.57$ ), MCS-26 (i.e., 64QAM with $R=0.64$ ), MCS-27 (i.e., 64QAM with $R=0.66$ ), and MCS-28 (i.e., 64QAM with $R=0.77$ ). Here, the UTW-OFDM-based LTE system with $N_{\mathrm{TR}} / N=0$ is equivalent to the conventional CPOFDM-based LTE system. Furthermore, $N_{\mathrm{TR}} / N=0.004$ is the minimum transition duration of the time-domain window.

In the case of MCS-9, the $E_{\mathrm{S}} / N_{0}$ to achieve BLER of $10^{-3}$ is not degraded from the conventional CP-OFDM with $N_{\mathrm{TR}} / N \leq 0.25$ and hardly deteriorated within $1.0 \mathrm{~dB}$ even with $N_{\mathrm{TR}} / N=1.0$. In the case of MCS-16, almost no BLER degradation is observed as well as QPSK when $N_{\mathrm{TR}} / N \leq 0.25$. As $N_{\mathrm{TR}} / N$ increases from 0.25 , the $E_{\mathrm{S}} / N_{0}$ to achieve BLER of $10^{-3}$ deteriorates, and an error floor is observed when $N_{\mathrm{TR}} / N=1.0$. In the case of MCS-22, 


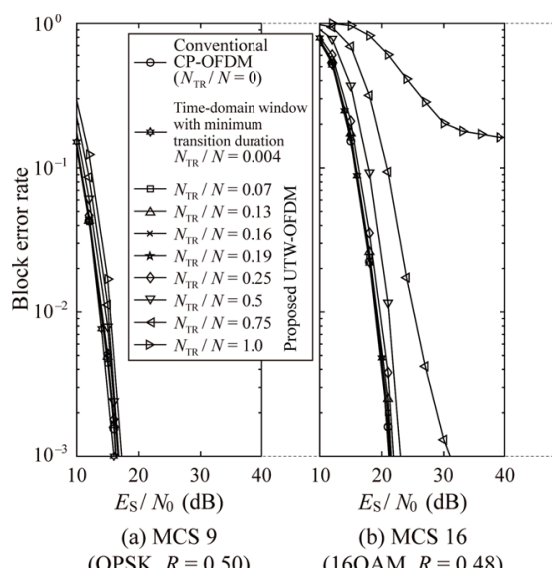

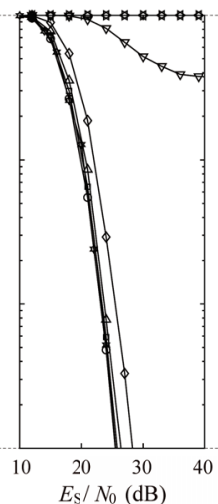

(c) $\operatorname{MCS} 22$

(64QAM, $R=0.48)$

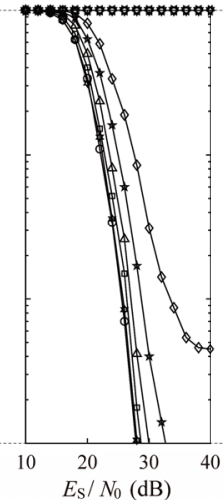

(d) $\operatorname{MCS} 24$

(16QAM, $R=0.57)$

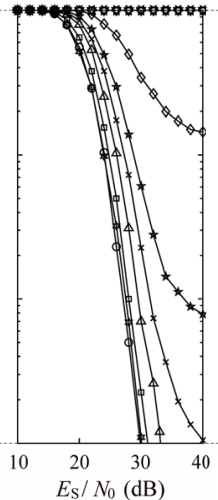

(e) MCS 26

(16QAM, $R=0.64)$

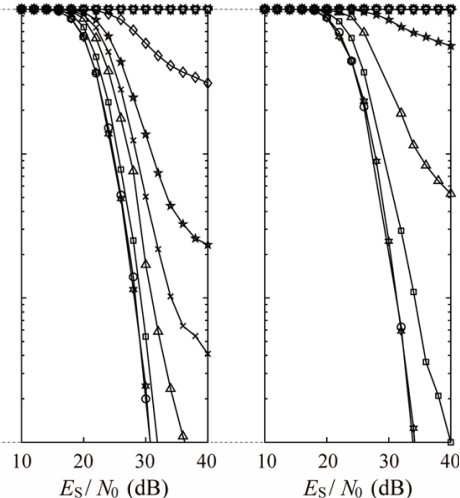

(g) MCS 28 (64QAM, $R=0.77$ )

Fig. 8 BLER of proposed UTW-OFDM-based LTE downlink system with variable UTW transition duration under EVA70 channel model: (a) MCS 9 (QPSK, $R=0.50$ ), (b) MCS 16 (16QAM, $R=0.48$ ), (c) MCS 22 (64QAM, $R=0.48$ ), (d) MCS 24 (64QAM, $R=0.57$ ), (e) MCS 26 (64QAM, $R=0.64$ ),

(f) MCS 27 (64QAM, $R=0.66$ ), (g) MCS 28 (64QAM, $R=0.77$ ).

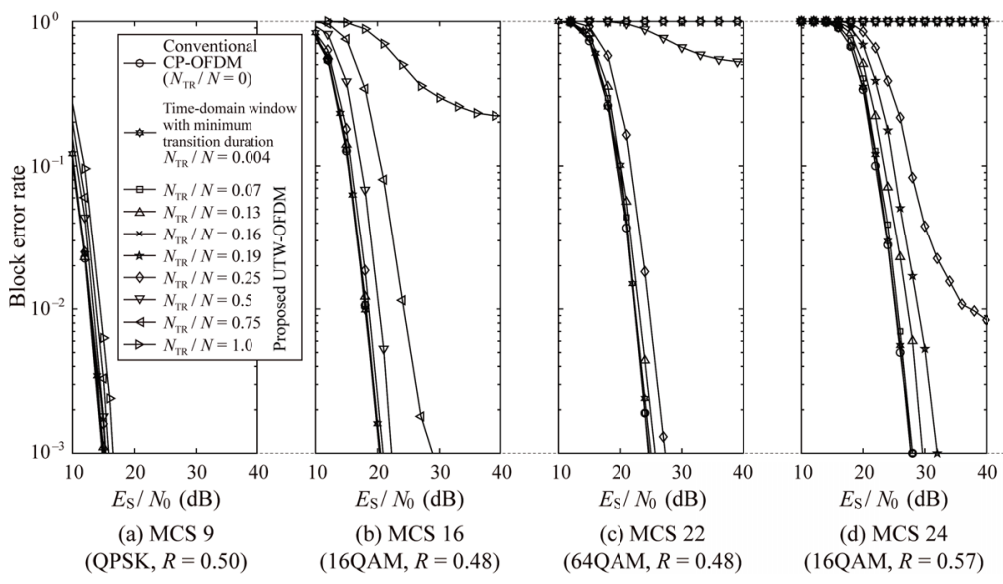

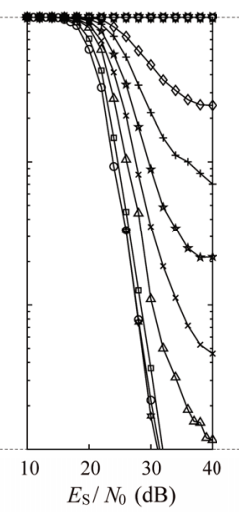

(e) MCS 26 (16QAM, $R=0.64)$

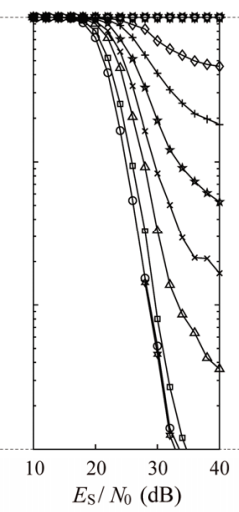

(f) MCS 27 (64QAM, $R=0.66$ )

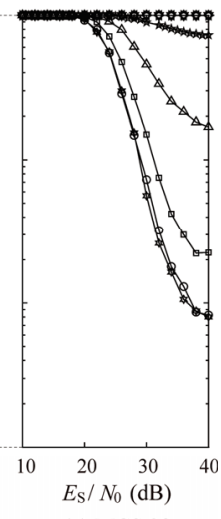

(g) MCS 28 (64QAM, $R=0.77$ )

Fig. 9 BLER of proposed UTW-OFDM-based LTE downlink system with variable UTW transition duration under ETU70 channel model: (a) MCS 9 (QPSK, $R=0.50$ ), (b) MCS 16 (16QAM, $R=0.48$ ), (c) MCS 22 (64QAM, $R=0.48$ ), (d) MCS 24 (64QAM, $R=0.57$ ), (e) MCS 26 (64QAM, $R=0.64$ ), (f) MCS 27 (64QAM, $R=0.66$ ), (g) MCS 28 (64QAM, $R=0.77$ ).

the $E_{\mathrm{S}} / N_{0}$ to achieve BLER of $10^{-3}$ is hardly deteriorated with $N_{\mathrm{TR}} / N \leq 0.13$ (i.e., 64/512). However, the $E_{\mathrm{S}} / N_{0}$ to achieve BLER of $10^{-3}$ deteriorates with $N_{\mathrm{TR}} / N>0.25$. Specifically, in the case of $N_{\mathrm{TR}} / N=0.25$, the $E_{\mathrm{S}} / N_{0}$ to achieve BLER of $10^{-3}$ is deteriorated by about $2.7 \mathrm{~dB}$. In the case of $N_{\mathrm{TR}} / N=0.5$, an error floor is observed. In the cases of MCS-24, MCS-26, and MCS-27, the $E_{\mathrm{S}} / N_{0}$ to achieve BLER of $10^{-3}$ are degraded by about $2.2 \mathrm{~dB}, 3.2 \mathrm{~dB}$, and $5.6 \mathrm{~dB}$, respectively, when $N_{\mathrm{TR}} / N$ is set to 0.13 . In the case of MCS-28, UTW transition duration with the condition of $N_{\mathrm{TR}} / N=0.07$ can achieve BLER of $10^{-3}$ with $E_{\mathrm{S}} / N_{0}$ degradation of $6.1 \mathrm{~dB}$, however, an error floor is observed when $N_{\mathrm{TR}} / N \geq 0.13$.

\subsubsection{Evaluation under ETU70 Channel Model}

Figure 9 shows the BLER characteristics of the proposed UTW-OFDM-based LTE downlink system with MCS-9,
MCS-16, MCS-22, MCS-24, MCS-26, MCS-27, and MCS28 under the 3GPP ETU70 channel model. In the case of MCS-9, the $E_{\mathrm{S}} / N_{0}$ to achieve BLER of $10^{-3}$ is slightly deteriorated only within $1.7 \mathrm{~dB}$ even with $N_{\mathrm{TR}} / N=1.0$. In the case of MCS-16, the $E_{\mathrm{S}} / N_{0}$ to achieve BLER of $10^{-3}$ is deteriorated by only $0.6 \mathrm{~dB}$ with $N_{\mathrm{TR}} / N=0.25$. However, the $E_{\mathrm{S}} / N_{0}$ to achieve BLER of $10^{-3}$ remarkably deteriorates with $N_{\mathrm{TR}} / N>0.5$, and an error floor is observed with $N_{\mathrm{TR}} / N=1.0$. In the case of MCS-22, the $E_{\mathrm{S}} / N_{0}$ to achieve BLER of $10^{-3}$ is hardly deteriorated with $N_{\mathrm{TR}} / N \leq 0.13$. However, the $E_{\mathrm{S}} / N_{0}$ to achieve BLER of $10^{-3}$ is deteriorated by about $2.8 \mathrm{~dB}$ with $N_{\mathrm{TR}} / N=0.25$, and an error floor is observed with $N_{\mathrm{TR}} / N \geq 0.5$. In the case of MCS-24, BLER of $10^{-3}$ can be achieved within a $4.0 \mathrm{~dB}$ degradation of the required $E_{\mathrm{S}} / N_{0}$ when $N_{\mathrm{TR}} / N \leq 0.19$. In the cases of MCS-26 and MCS-27, the $E_{\mathrm{S}} / N_{0}$ to achieve BLER of $10^{-3}$ are degraded by within about $0.5 \mathrm{~dB}$ and $1.2 \mathrm{~dB}$, respectively, when $N_{\mathrm{TR}} / N \leq 0.07$. In the case of MCS-28, 
the UTW transition duration with any conditions of $N_{\mathrm{TR}} / N$ cannot achieve BLER of $10^{-3}$.

\section{Discussion}

From the results in Sect. 4, the proposed UTW-OFDM-based LTE downlink system achieves sufficient OOBE reduction, and the turbo coding scheme can compensate the waveform distortion to some extent caused by long UTW transition duration. To confirm the feasibility and practicality of the proposed UTW-OFDM, we clarify how far OOBE can be reduced without the degradation of communication quality in this section. Furthermore, we discuss the implementation complexity of the proposed UTW-OFDM with respect to the number of required multiplications.

5.1 Potential of OOBE Reduction without BLER Degradation

To confirm the potential of the proposed UTW-OFDM, we first discuss the trade-off between the OOBE reduction and the BLER. Figures 10(a) and (b) show the relationship between the averaged PSD at the channel-edge (i.e., OOBE reduction performance) and the $E_{\mathrm{S}} / N_{0}$ to achieve BLER of $10^{-1}$ with various $N_{\mathrm{TR}}$ evaluated under the $3 \mathrm{GPP}$ EVA70 and ETU70 channel models, respectively. The characteristics under EVA70 and ETU70 show almost the same tendency for the change of the $N_{\mathrm{TR}} / N$ in each MCS. In the case of MCS9, the proposed UTW-OFDM can reduce OOBE by about $45 \mathrm{~dB}$ without BLER degradation compared to the conventional CP-OFDM. When an about $2.0 \mathrm{~dB}$ degradation of the $E_{\mathrm{S}} / N_{0}$ to achieve BLER of $10^{-1}$ is acceptable, the proposed UTW-OFDM can reduce OOBE by about $68 \mathrm{~dB}$. In the case of MCS-16, when an about $0.5 \mathrm{~dB}$ degradation of the $E_{\mathrm{S}} / N_{0}$ to achieve BLER of $10^{-1}$ is acceptable, the proposed UTWOFDM can reduce OOBE by about $45 \mathrm{~dB}$. Furthermore, when an about $2.0 \mathrm{~dB}$ degradation of the $E_{\mathrm{S}} / N_{0}$ to achieve BLER of $10^{-1}$ is acceptable, the proposed UTW-OFDM can reduce OOBE by about $56 \mathrm{~dB}$. In the case of MCS-22, when an about $0.5 \mathrm{~dB}$ degradation of the $E_{\mathrm{S}} / N_{0}$ to achieve BLER of $10^{-1}$ is acceptable, the proposed UTW-OFDM can reduce OOBE by about $32 \mathrm{~dB}$. Furthermore, when an about $2.0 \mathrm{~dB}$ degradation of the $E_{\mathrm{S}} / N_{0}$ to achieve BLER of $10^{-1}$ is acceptable, the proposed UTW-OFDM can reduce OOBE by about $45 \mathrm{~dB}$. As the coding rate increases, the influence of the ISI and ICI due to the long UTW transition duration also increases, resulting in the degradation of communication quality or the limitation of the OOBE reduction performance of the proposed UTW-OFDM. Specifically, for when MCS-26, the performance of the OOBE reduction is limited to $40 \mathrm{~dB}$, and then it is required to allow about $5 \mathrm{~dB}$ degradation of the $E_{\mathrm{S}} / N_{0}$ to achieve BLER of $10^{-1}$.

Figures 11(a) and (b) show the relationship between the averaged PSD at the channel-edge and the $E_{\mathrm{S}} / N_{0}$ to achieve BLER of $10^{-3}$ with various $N_{\text {TR }}$ evaluated under the $3 \mathrm{GPP}$ EVA70 and ETU70 channel models, respectively. The characteristics under EVA70 and ETU70 show almost the same

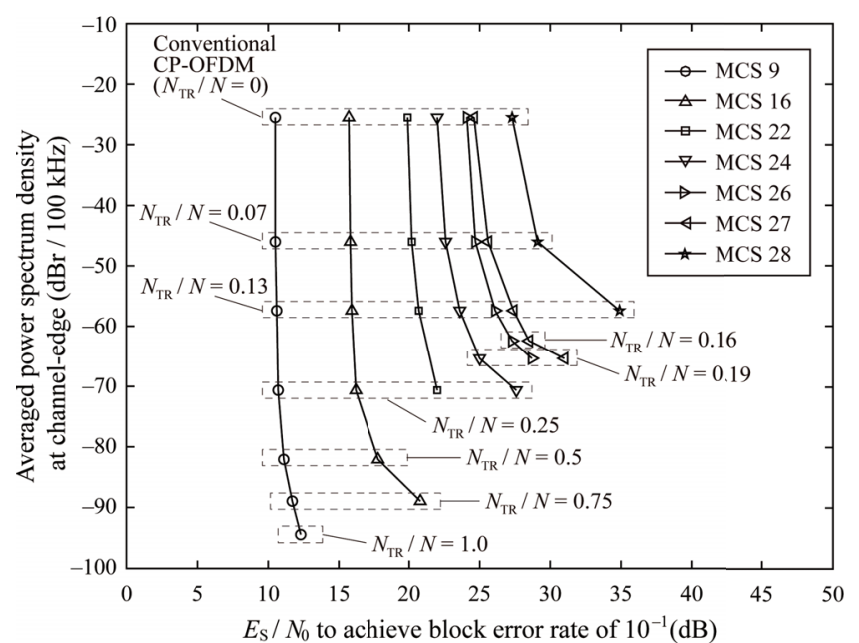

(a) EVA70

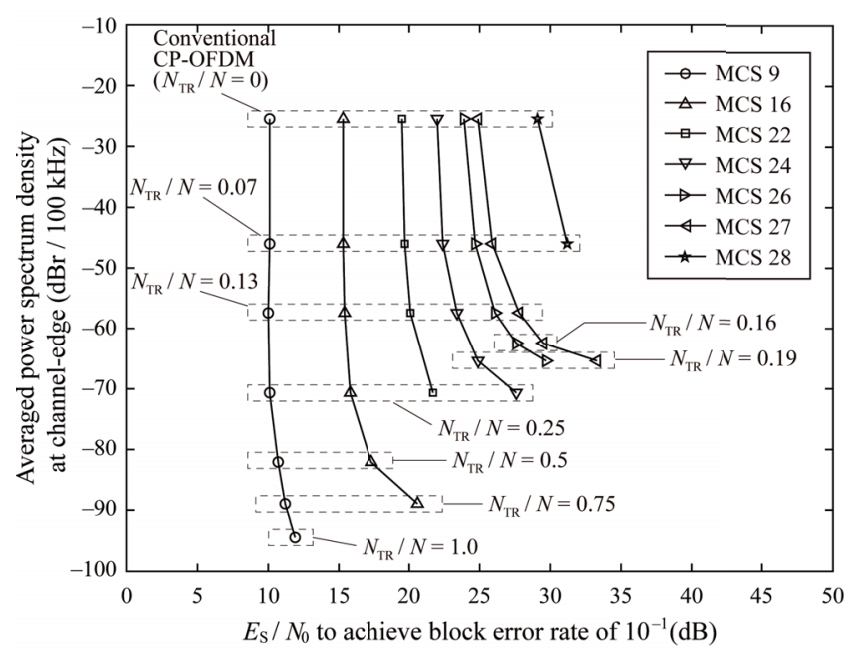

(b) ETU70

Fig. 10 OOBE reduction performance vs $E_{\mathrm{S}} / N_{0}$ to achieve BLER of $10^{-3}$.

tendency for the change of the $N_{\mathrm{TR}} / N$ in each MCS. In the case of MCS-9, when an about $0.5 \mathrm{~dB}$ or $2.0 \mathrm{~dB}$ degradation of the $E_{\mathrm{S}} / N_{0}$ to achieve BLER of $10^{-3}$ is acceptable, the proposed UTW-OFDM can reduce OOBE by about $45 \mathrm{~dB}$ or $68 \mathrm{~dB}$, respectively, compared to the conventional CPOFDM. In the case of MCS-16, when an about $0.5 \mathrm{~dB}$ or $2.0 \mathrm{~dB}$ degradation of the $E_{\mathrm{S}} / N_{0}$ to achieve BLER of $10^{-3}$ is acceptable, the proposed UTW-OFDM can reduce OOBE by about $45 \mathrm{~dB}$ or $56 \mathrm{~dB}$, respectively. In the case of MCS-22, when an about $1.0 \mathrm{~dB}$ or $3.0 \mathrm{~dB}$ degradation of the $E_{\mathrm{S}} / N_{0}$ to achieve BLER of $10^{-3}$ is acceptable, the proposed UTWOFDM can reduce OOBE by about $32 \mathrm{~dB}$ or $45 \mathrm{~dB}$, respectively. As the coding rate increases, the influence of the ISI and ICI due to the long UTW transition duration increases, resulting in the degradation of communication quality or the limitation of the OOBE reduction performance of the proposed UTW-OFDM. Specifically, for when MCS-26, the performance of the OOBE reduction is limited to $40 \mathrm{~dB}$, and then it is required to allow about $5 \mathrm{~dB}$ degradation of the $E_{\mathrm{S}} / N_{0}$ to achieve BLER of $10^{-3}$. 


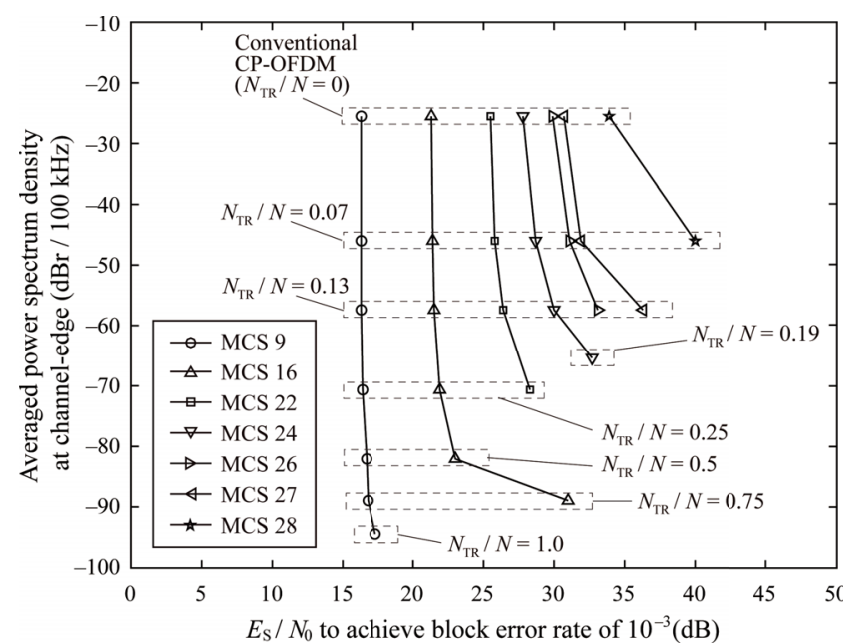

(a) EVA70

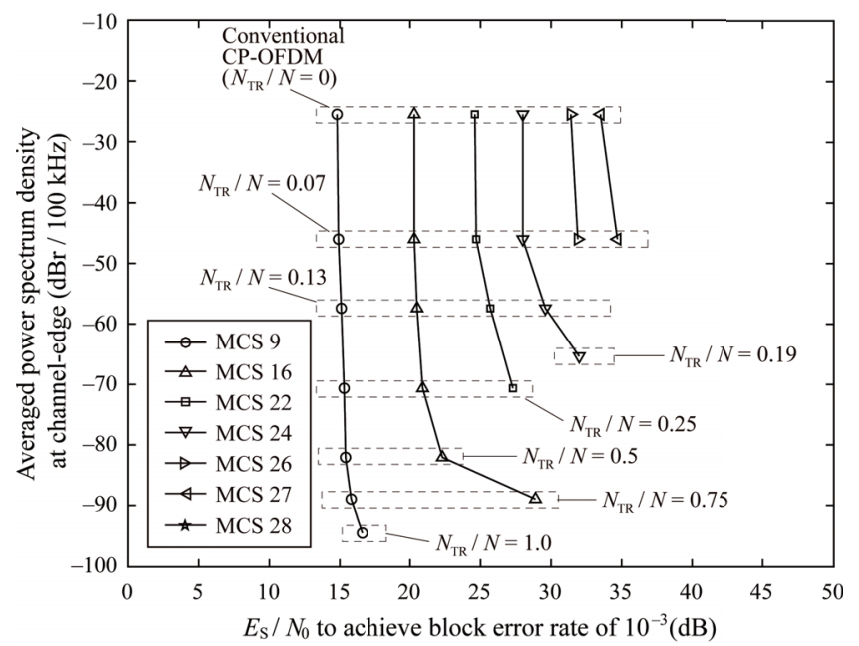

(b) ETU70

Fig. 11 OOBE reduction performance vs $E_{S} / N_{0}$ to achieve BLER of $10^{-1}$.

From these results, when the half coding rate is applied, the proposed UTW-OFDM significantly reduces OOBE without BLER degradation compared to the conventional CP-OFDM for any modulation scheme, even though the propagation channel has long multi-path delay exceeding the $\mathrm{CP}$ length. On the other hand, when the higher coding rate is applied with 64QAM, the OOBE reduction performance of the proposed UTW-OFDM gradually decreases. Therefore, the advantage of the proposed UTW-OFDM appears prominently when applying lower MCS level under the low $E_{\mathrm{S}} / N_{0}$ environment.

\subsection{Complexity}

As described in Sect. 3, the proposed UTW-OFDM has highcompatibility with the conventional CP-OFDM because only two multiplications (and a storage memory of the UTW coefficient) are additionally required to the transmitter. In this section, we discuss the implementation complexity by calculating and comparing the required number of multiplications

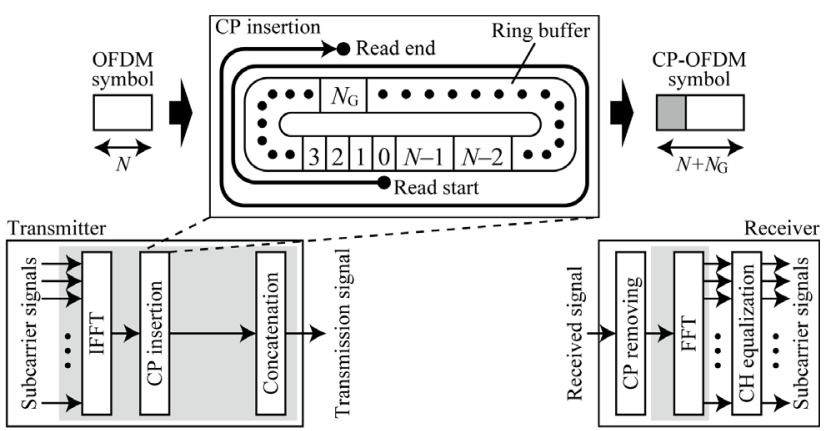

Fig. 12 Transceiver architecture of conventional CP-OFDM-based LTE downlink system.

for the conventional CP-OFDM, the proposed UTW-OFDM, and the UF-OFDM (as a typical filtering-based OFDM). Note that these schemes differ in the implementation complexity but achieve equivalent throughput in environments with adequate $E_{\mathrm{S}} / N_{0}$. In this evaluation, the number of required complex multiplications is used as metrics of implementation complexity and calculated for the IFFT (or IDFT) in the encoder, the FFT (or DFT) in the decoder, and additional processing (i.e., windowing or filtering) individually.

\subsubsection{Conventional CP-OFDM-Based System}

A typical transceiver architecture of the conventional CPOFDM-based LTE downlink system is depicted in Fig. 12. The $N$-point IFFT and the $N$-size ring buffer are implemented in the transmitter, and the $\mathrm{CP}$ is inserted by using the ring buffer. In the receiver, the $N$-point FFT is implemented. When the Cooley-Tukey algorithm (Radix-2 FFT) [27] is applied, the $N$-point IFFT and the $N$-point FFT require $(N / 2) \log _{2} N$ complex multiplications.

\subsubsection{UTW-OFDM-Based System}

Figure 13 shows a transceiver architecture of the UTWOFDM-based LTE downlink system. In the transmitter, the $\mathrm{OM}$ insertion and the windowing process are added to the conventional CP-OFDM transmitter. The OM insertion can be performed by extending the $\mathrm{CP}$ insertion process. When the read start index of the ring buffer is changed from 0 to $\left(N-N_{\mathrm{M}}-1\right)$, the suffix-OM of $N_{\mathrm{M}}+1$ sample points can be inserted. Similarly, when the read end index of the ring buffer is changed from $N_{\mathrm{G}}$ to $\left(N_{\mathrm{G}}+N_{\mathrm{M}}\right)$, the prefixOM of $N_{\mathrm{M}}$ sample points can be prefixed to the CP of $N_{\mathrm{G}}$ sample points. Therefore, the $\mathrm{OM}$ insertion processing by no means increases any complexity compared with the conventional CP-OFDM-based transmitter. An additional windowing process requires only one multiplication to multiple the UTW function, while two windowing processors (i.e., two additional multiplications) are necessary to overlap successive symbols in real time. Therefore, $2+(N / 2) \log _{2} N$ complex multiplications are required in the transmitter. On the other hand, since the conventional CP-OFDM receiver can be applied to the UTW-OFDM-based LTE downlink sys- 


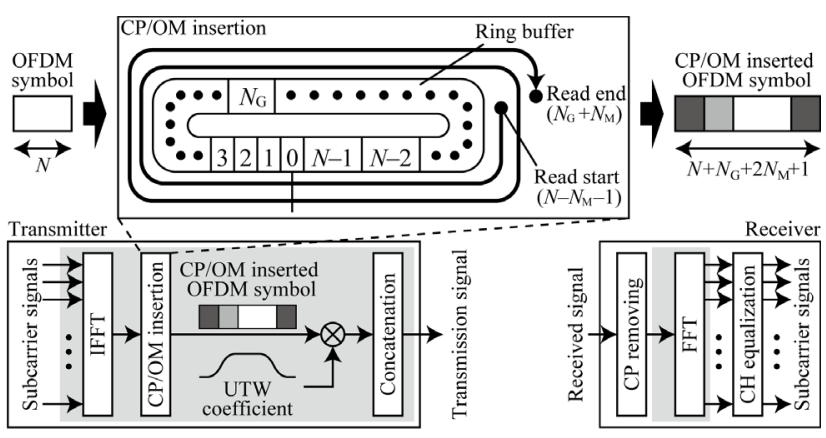

Fig. 13 Transceiver architecture of UTW-OFDM-based LTE downlink system.

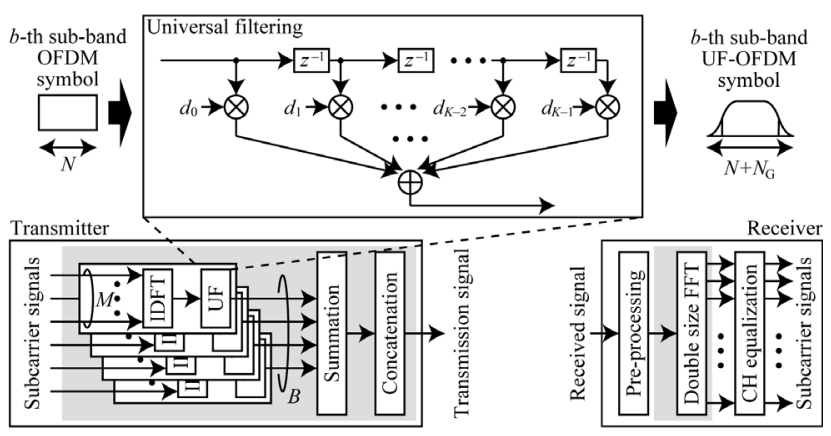

Fig. 14 Transceiver architecture of UF-OFDM-based LTE downlink system.

tem, the $(N / 2) \log _{2} N$ complex multiplications are required in the receiver as well as the CP-OFDM.

\subsubsection{UF-OFDM-Based System}

Figure 14 shows a transceiver architecture of the UF-OFDMbased LTE downlink system [6]-[8]. In the transmitter, subcarriers in the transmission band are firstly grouped into $B$ sub-bands, and each sub-band contains $M$ subcarriers. In each sub-band, $M$ subcarriers are converted to the sub-band OFDM symbol by using the $M-N$-point IDFT. Then, each sub-band OFDM symbol is converted to the sub-band UFOFDM symbol with the $K$-taps universal filter. Finally, the sub-band UF-OFDM symbols are summed up to generate the UF-OFDM symbol. The $M-N$-point IDFT in each sub-band has $M N$ complex multiplications and thus, $B M N$ complex multiplications are totally required for the IDFTs. Furthermore, the $K$-taps universal filter in each sub-band has $K$ complex multiplications, and $B K$ complex multiplications are required for the universal filter process. In the receiver, a double-size FFT (i.e., $2 N$-point FFT) that requires $N \log _{2}(2 N)$ complex multiplications is used to obtain subcarrier signals from the received UF-OFDM symbol.

\subsubsection{Complexity Evaluation with LTE Downlink Param- eters}

The required number of complex multiplications for each transceiver is summarized in Table 4. Figure 15 shows the
Table 4 Number of complex multiplications for transceivers.

\begin{tabular}{l|l|l}
\hline \multirow{2}{*}{ Waveforms } & \multicolumn{2}{|l}{ Number of complex multiplications } \\
\cline { 2 - 3 } & Transmitter & Receiver \\
\hline CP-OFDM & $(N / 2) \log _{2} N$ & $(N / 2) \log _{2} N$ \\
\hline UTW-OFDM & $(N / 2) \log _{2} N+2$ & $(N / 2) \log _{2} N$ \\
\hline UF-OFDM & $B M N+B K$ & $N \log _{2}(2 N)$ \\
\hline
\end{tabular}

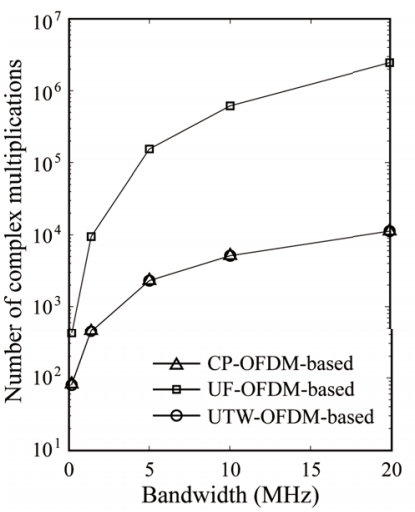

(a) Transmitter

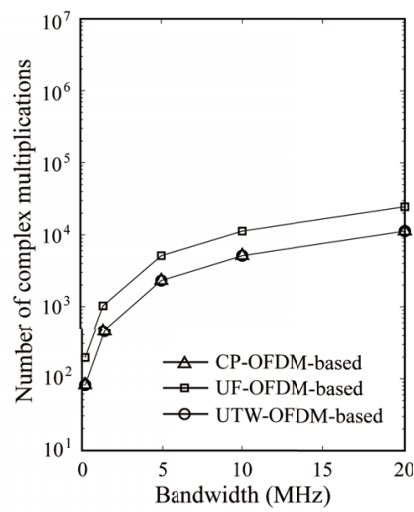

(b) Receiver
Fig. 15 Number of complex multiplications for transmitter and receiver of CP-OFDM-, UF-OFDM-, and UTW-OFDM-based LTE downlink systems.

Table 5 Parameter sets of $\{N, B, M, K\}$.

\begin{tabular}{l|l|l|l|l}
\hline Bandwidth & $N$ & $B$ & $M$ & $K$ \\
\hline $180 \mathrm{kHz}$ & 32 & 1 & 12 & 37 \\
\hline $1.4 \mathrm{MHz}$ & 128 & 6 & 12 & 37 \\
\hline $5 \mathrm{MHz}$ & 512 & 25 & 12 & 37 \\
\hline $10 \mathrm{MHz}$ & 1024 & 50 & 12 & 37 \\
\hline $20 \mathrm{MHz}$ & 2048 & 100 & 12 & 37 \\
\hline
\end{tabular}

calculated number of complex multiplications for the conventional CP-OFDM, the proposed UTW-OFDM, and the UF-OFDM systems by using the $200 \mathrm{kHz}$ bandwidth NBIoT parameters and $1.4 \mathrm{MHz}, 5 \mathrm{MHz}, 10 \mathrm{MHz}$, and $20 \mathrm{MHz}$ bandwidth LTE downlink parameters shown in Table 5.

First, we discuss the complexity of the transmitter with Fig. 15(a). Regardless of the bandwidth, the complexity of the UTW-OFDM transmitter is almost the same as that of the CP-OFDM transmitter. When the bandwidth is $5 \mathrm{MHz}$, the required number of complex multiplications in the conventional CP-OFDM transmitter and the UTW-OFDM transmitter is 2,304 and 2,306, respectively. In contrast, the required number of complex multiplications in the UF-OFDM transmitter comes to 154,525 due to the multiple DFTs and a large number of filter taps. As a result, the complexity of the UFOFDM transmitter increases by about 67 times compared to the UTW-OFDM. As the bandwidth increases, the difference of the required number of complex multiplications in the UTW-OFDM transmitter and the UF-OFDM transmitter becomes large. When the bandwidth is $20 \mathrm{MHz}$, the complexity of the UF-OFDM transmitter increases by about two hundred times compared to the UTW-OFDM. On the other hand, as the bandwidth decreases, the difference of the required number of complex multiplications in the UTWOFDM transmitter and the UF-OFDM transmitter becomes 
small. However, there still has a five-fold difference when the bandwidth is $200 \mathrm{kHz}$.

Second, we discuss the complexity of the receiver with Fig. 15(b). Regardless of the bandwidth, the UF-OFDM receiver almost doubles the complexity compared to the $\mathrm{CP}$ OFDM and the UTW-OFDM due to the double-size FFT. When the bandwidth is $5 \mathrm{MHz}$, the required number of complex multiplications in the conventional CP-OFDM receiver and the UTW-OFDM receiver is 2,304. On the other hand, the required number of complex multiplications in the UFOFDM receiver comes to 5,120. From the viewpoint of the number of complex multiplications, the UTW-OFDM-based system is more compatible with the conventional CP-OFDMbased system than the UF-OFDM-based system.

\section{Conclusion}

In this paper, the performance of the proposed UTW-OFDMbased LTE downlink system is comprehensively evaluated to confirm its feasibility and practicality for the $5 \mathrm{G}$ and beyond from the aspects of OOBE reduction performance, BLER characteristics, and implementation complexity. Under the channel models of 3GPP EVA70 and 3GPP ETU70, the proposed UTW-OFDM reduces OOBE by $45 \mathrm{~dB}$ with only $2.0 \mathrm{~dB}$ degradation of the $E_{\mathrm{S}} / N_{0}$ to achieve BLER $=10^{-1}$ compared to the conventional CP-OFDM, even if 64QAM with a half coding rate is applied. Furthermore, the UTWOFDM-based system has almost the same complexity as the conventional CP-OFDM-based system (which is low), and so is much less complex than the UF-OFDM-based system. These results demonstrate that the proposed UTW-OFDM is a practical waveform applicable to $5 \mathrm{G}$ and beyond. In the future work, we will evaluate the performance of the proposed UTW-OFDM applying the low-density parity-check (LDPC) coding, which is a more powerful channel coding technique commonly used in the $5 \mathrm{G}$.

\section{Acknowledgments}

A part of this research was conducted under a contract of R\&D for Expansion of Radio Wave Resources, organized by the Ministry of Internal Affairs and Communications, Japan. And, a part of this work was supported by JSPS KAKENHI Grant Number 15K18064.

\section{References}

[1] International Telecommunications Union, "IMT Vision - Framework and Overall Objectives of the Future Development of IMT for 2020 and Beyond," Recommendation ITU-R M.2083-0, 2015.

[2] ETSI, "ETSI TS 136.101 V13.4.0 (2016-09)," Sept. 2016.

[3] B. Farhang-Boroujeny, "OFDM versus filter bank multicarrier," IEEE Signal Process. Mag., vol.28, no.3, pp.92-112, May 2011

[4] Y. Medjahdi, D. le Ruyet, F. Bader, and L. Martinod, "Integrating LTE broadband system in PMR band: OFDM vs. FBMC coexistence capabilities and performances," Proc. ISWCS2014, Aug. 2014.

[5] V. Vakilian, T. Wild, F. Schaich, S. ten Brink, and J.-F. Frigon, "Universal-filtered multi-carrier technique for wireless systems beyond LTE," Proc. IEEE Globecom Workshops (GC Wkshps), pp.223-228, Dec. 2013.

[6] J. Abdoli, M. Jia, and J. Ma, "Filtered OFDM: A new waveform for future wireless systems," Proc. IEEE SPAWC2015, pp.66-70, June 2015

[7] F. Schaich, T. Wild, and Y. Chen, "Waveform contenders for 5G - Suitability for short packet and low latency transmissions," Proc. IEEE VTC2014-Spring, pp.1-5, May 2014.

[8] M. Kibria, G. Villardi, K. Ishizu, and F. Kojima, "Throughput enhancement of multicarrier cognitive M2M networks: Universalfiltered OFDM systems," IEEE Internet Things J., vol.3, no.5, pp.830-838, Oct. 2016.

[9] C. Muschallik, "Improving an OFDM reception using an adaptive Nyquist windowing," IEEE Trans. Consum. Electron., vol.42, no.8, pp.259-269, Aug. 1996.

[10] T. Onizawa, M. Mizoguchi, M. Morikura, and T. Tanaka, "A fast synchronization scheme of OFDM signals for high-rate wireless LAN," IEICE Trans. Commun., vol.E82-B, no.2, pp.455-463, Feb. 1999.

[11] IEEE Std 802.11 ${ }^{\mathrm{TM}}-2016$, Dec. 2016.

[12] K. Mizutani, Z. Lan, and H. Harada, "Time-domain windowing design for IEEE 802.11 af based TVWS-WLAN systems to suppress out-of-band emission," IEICE Trans. Commun., vol.E97-B, no.4, pp.875-885, April 2014.

[13] R. Funada, K. Mizutani, and H. Harada, "Wireless transmitter, wireless receiver, wireless transmission method, and wireless reception method," Patent no.: US 9,450,800 B2, Sept. 20, 2016 (Submission Jan. 2013).

[14] FCC, "Unlicensed Operation in the TV Broadcast Bands," Third Memorandum Opinion and Order, FCC. 12-36, April 2012.

[15] K. Mizutani and H. Harada, "Universal time-domain windowed OFDM,” Proc. IEEE VTC2016-Fall, pp.1-5, Sept. 2016.

[16] K. Mizutani, T. Matsumura, and H. Harada, "A comprehensive study of universal time-domain windowed OFDM-based LTE downlink system," Proc. WPMC2017, pp.1-7, Dec. 2017.

[17] 3GPP, “3GPP TS 36.101 V8.27.0 (2016-03),” March 2016.

[18] Qualcomm Inc., "Waveform Candidates," 3GPP TSG-RAN WG1 \#84b, R1-162199, April 2016.

[19] S.Y. Chang, "Spectra and bandwidth overhead with and without Filtering for TG4m OFDM,” Doc.: IEEE802.15-12-0377-00-004m, July 2012.

[20] 3GPP, "3GPP TR 37.976 V11.0.0 (2012-3)," March 2012.

[21] 3GPP, “3GPP TS 36.211 V8.9.0 (2009-12)," Dec. 2009.

[22] 3GPP, “3GPP TS 36.213 V8.8.0 (2009-10)," Oct. 2009.

[23] J.C. Ikuno, M. Wrulich, and M. Rupp, "System level simulation of LTE networks," Proc. IEEE VTC2010-Spring, pp.1-5, May 2010.

[24] A. Duran, M. Toril, F. Ruiz, and A. Mendo, "Self-optimization algorithm for outer loop link adaptation in LTE," IEEE Commun. Lett., vol.19, no.11, pp.2005-2008, Nov. 2015.

[25] Investigation Committee on Technical Conditions for Expanding Use of Public Broadband Systems, "A Study on Technical Conditions for Expanding Use of Public Broadband Systems," Report of FY2016, March 2017 (in Japanese).

[26] K. Mizutani, H. Kuriki, Y. Kodama, T. Matsumura, and H. Harada, "Performance evaluation of TD-LTE in VHF-band for large coverage public broadband communications system," Proc. WPMC2017, pp.80-86, Dec. 2017.

[27] J. Whelchel and D. Guinn, "FFT organizations for high-speed digital filtering," IEEE Trans. Audio Electroacoust., vol.18, no.2, pp.159168, June 1970 


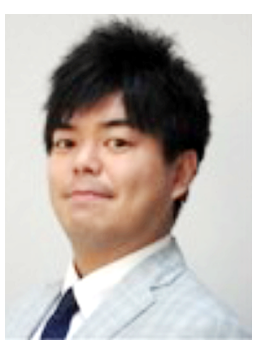

Keiichi Mizutani is an assistant professor of Kyoto University. He received a B.E. degree in electric, electrical and system engineering from the Osaka Prefecture University, Japan, in 2007, and an M.E. and Ph.D. degree in electric and electrical engineering from the Tokyo Institute of Technology, Japan, in 2009 and 2012 respectively. He was an invited researcher at Fraunhofer Heinrich Hertz Institute, Germany, in 2010. From April 2012 to Sept. 2014, he was a researcher at National Institute of Information and Communications Technology (NICT). He currently researches the topics of physical layer technologies in White Space Communications, Dynamic Spectrum Access, Wireless Smart Utility Networks (Wi-SUN), and the $4 \mathrm{G}$ and $5 \mathrm{G}$ access technologies including OFDM, OFDMA, MIMO, and multi-hop relay network systems. Since joining in NICT, he had been involved in IEEE 802 standardization activities, namely 802.11 af, $802.15 .4 \mathrm{~m}$ and 802.22b. He received the Special Technological Award and Best Paper Award from IEICE SR technical committee in 2009 and 2010 respectively, the Young Researcher's Award from IEICE SRW technical committee in 2016, and WPMC 2017 Best Paper Award. Dr. Mizutani is a member of the IEEE.

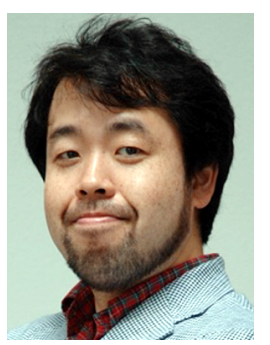

Takeshi Matsumura received the M.S. degrees in Electronic Engineering in 1998 and Ph.D. in Nano-mechanics Engineering in 2010, from Tohoku University. During 1998-2007, he had been engaged in the $R \& D$ of wireless communications devices in some companies. In April 2007, he joined National Institute of Information and Communications Technology (NICT) as a researcher in the Smart Wireless Laboratory and engaged in the white-space communication systems and 5th generation mobile communication systems. Since April 2016, he has been an associated professor of Graduate School of Informatics, Kyoto University and he has been also a senior researcher in Wireless Systems Laboratory in Wireless Networks Research Center at NICT. His research interests include white-space communication systems, wide-area wireless network systems and 5th generation mobile communication systems. Dr. Matsumura is a member of the IEEE.

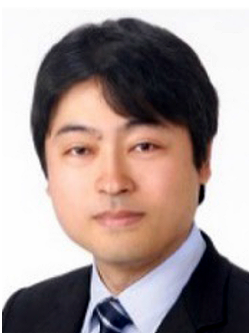

Hiroshi Harada is a professor of Graduate School of Informatics, Kyoto University, and an executive research director of Social ICT Research Center at National Institute of Information and Communications Technology (NICT). He joined the Communications Research Laboratory, Ministry of Posts and Communications, in 1995 (currently NICT). Since 1995, he has researched software defined radio (SDR), $\operatorname{cog}$ nitive radio, dynamic spectrum access network, wireless smart utility network (Wi-SUN), and broadband wireless access systems on the microwave and millimeter-wave band. He also has joined many standardization committees and forums in United States as well as in Japan and fulfilled important roles for them, especially IEEE 1900 and IEEE 802 . He serves currently on the board of directors of Wi-SUN Alliance and WhiteSpace Alliance and served as the chair of IEEE DySpan standards committee and the vice chair of IEEE P1900.4, P802.15.4g, P802.15.4m, and TIA TR-51. He moreover was the chair of the IEICE Technical Committee on Software Radio (TCSR) in 2005-2007. $\mathrm{He}$ also is involved in many other activities related to telecommunications. He was a visiting professor of the University of Electro-Communications, Tokyo, Japan in 2005-2014, and is the author of Simulation and Software Radio for Mobile Communications (Artech House, 2002). 